\title{
Research on System Dynamics Model of Urban Environment Livability Evaluation
}

\author{
Hongxue $\mathrm{Xu}$ \\ School of Information Engineering \\ Shenyang University \\ Shenyang, China \\ hongxuexu@163.com
}

\author{
Mingtong Xu \\ Development department \\ Baidu.com \\ Beijing, China \\ 372943817@qq.com
}

\begin{abstract}
Focus on the livability evaluation problem of urban environment, based on the system dynamics theory and the system analysis method, through research and analysis on the evaluation index system quantitative relation of urban livable environment, build an urban environment livability evaluation system dynamics model, and use the parameter estimation method to test the effectiveness of the model, and based on the utility function, through analysis on the quantitative relationship of the model variables, a parameter estimation algorithm of livability utility function is given, it is used to determine the livability evaluation algorithm of urban environment.
\end{abstract}

Keywords-urban environment; livability evaluation; system dynamics

\section{INTRODUCTION}

Urban livable environment problems is a system problems that involving a wide range and perplexing relationship [1]. Urban livable environment system includes the various factors of natural environment and social environment [2]. In order to facilitate the livability evaluation of urban environment, it is required to build a livability evaluation system dynamics model of urban environment.

In this paper, based on the evaluation index system of livable city [3], through research and analysis on the evaluation index system quantitative relation of urban environment, determine the main factors which impact the livability evaluation results of urban environment.

Using system analysis method to build livability evaluation system dynamics model of urban environment, and the parameter estimation method is used to determine the model variables.

And combined with effective data provide statistical yearbook of Shenyang Tiexi District [4], testing the effectiveness of the model with the method of system simulation, test results show that the model is effective.

\section{LIVABILITY EVALUATION MODEL OF URBAN ENVIRONMENT}

According to the theory and method of system dynamics [5] to build the livability evaluation system dynamics relationship of urban environment. The mathematical relationship of related index quantity is described below.

\section{A. Basic survival environment livability fuction expressions}

The basic survival environment livability fuction expressions include: $Z 1=f($ Time $) ; L Z 1=f(Z 1) ; Z 2=f($ Time $)$; LZ2 = f(Z2); Z3 = f(Time); LZ3 = f(Z3); Z4 = f(Time); LZ4 = $f(Z 4) ; Z 5=f($ Time $) ; L Z 5=f(Z 5) ; Z 6=f($ Time $) ; L Z 6=f(Z 4)$; $L Z=f(L Z 1, L Z 2, L Z 3, L Z 4, L Z 5, L Z 6)$.

$Z 1$ represents the compliance rate of noise environmental quality function area, $L Z 1$ represents the compliance rate of noise environmental quality function area livability, Z2 represents the coverage rate of noise compliance area, $L Z 2$ represents the coverage rate of noise compliance area livability, Z3 represents the compliance rate of water environmental quality function area, LZ3 represents the compliance rate of water environmental quality function area livability, Z4 represents the water pollution index, LZ4 represents the water pollution index livability, $Z 5$ represents the compliance rate of air environmental quality function area, $L Z 5$ represents the compliance rate of air environmental quality function area livability, $Z 6$ represents the air pollution index, LZ6 represents the air pollution index livability, $L Z$ represents the basic survival environment livability.

\section{B. Soil environment protection livability fuction expressions}

The soil environment protection livability fuction expressions include: $O 1=f($ Time $) ; L O 1=f(O 1) ; 02=$ $f($ Time $) ; L O 2=f(O 2) ; O 3=f($ Time $) ; L O 3=f(O 3) ; L O=$ $f(L O 1, L O 2, L O 3)$.

O1 represents the residues rate of industrial waste in soil, LO1 represents the residues rate of industrial waste in soil livability, $\mathrm{O} 2$ represents the detection rate of harmful toxic substances in the soil, $\mathrm{LO} 2$ represents the detection rate of harmful toxic substances in the soil livability, O3 represents the excessive rate of heavy metal in soil, $L O 3$ represents the excessive rate of heavy metal in soil livability, $L O$ represents the soil environment protection livability.

\section{Waste disposal and environmental pleasant degree livability fuction expressions}

The waste disposal and environmental pleasant degree livability fuction expressions include: $R 1=f($ Time $) ; L R 1=$ $f(R 1) ; R 2=f($ Time $) ; L R 2=f(R 2) ; R 3=f($ Time $) ; L R 3=f(R 3)$; $L R=f(L R 1, L R 2, L R 3) ; I 1=f(L Z, L R, L O)$.

$R 1$ represents the "Three wastes" comprehensive disposal rate, LR1 represents the "Three wastes" comprehensive 
disposal rate livability, $R 2$ represents the repeat utilization rate of industrial water, $L R 2$ represents the repeat utilization rate repeat utilization rate of industrial water livability, $R 3$ represents the cyclic utilization rate of living garbage, $L R 3$ represents the cyclic utilization rate of living garbage livability, $L R$ represents the waste disposal livability, I1 represents the environmental pleasant degree livability.

\section{Landscape protection and landscape beautiful degree livability fuction expressions}

The landscape protection and landscape beautiful degree livability fuction expressions include: $G 1=f($ Time $)$; $L G 1=$ $f(G 1) ; G 2=f($ Time $) ; L G 2=f(G 2) ; G 3=f($ Time $) ; L G 3=f(G 3)$; $G 4=f($ Time $) ; L G 4=f(G 4) ; L G=f(L G 1, L G 2, L G 3, L G 4) ; V 1$ $=f($ Time $) ; L V 1=f(V 1) ; V 2=f($ Time $) ; L V 2=f(V 2) ; V 3=$ $f($ Time $) ; L V 3=f(V 3) ; L V=f(L V 1, L V 2, L V 3) ; I 2=f(L G, L V)$.

$G 1$ represents the the urban vegetation coverage rate, $L G 1$ represents the the urban vegetation coverage rate livability, G2 represents the urban waters coverage rate, $L G 2$ represents the urban waters coverage rate livability, G3 represents the number of the urban park, $L G 3$ represents the number of the urban park livability, $G 4$ represents the number of the urban square, $L G 4$ represents the number of the urban square livability, $L G$ represents the natural landscape livability, $V 1$ represents the humanities landscape retention rate, $L V 1$ represents the humanities landscape retention rate livability, $V 2$ represents the humanities landscape intact rate, $L V 2$ represents the humanities landscape intact rate livability, V3 represents the humanities landscape protection public participation rate, $L V 3$ represents the humanities landscape protection public participation rate livability, $L V$ represents the humanities landscape livability, I2 represents the landscape protection and landscape beautiful degree livability.

\section{E. Basic service facilities livability fuction expressions}

The basic service facilities livability fuction expressions include: $S 1=\mathrm{F}($ Time $) ; L S 1=\mathrm{F}(S 1) ; S 2=f($ Time $) ; L S 2=f(S 2)$; $S 3=f($ Time $) ; L S 3=f(S 3) ; S 4=f($ Time $) ; L S 4=f(S 4) ; S 5=$ $f($ Time $) ; L S 5=f(S 5) ; S 6=f($ Time $) ; L S 6=f(S 6) ; S 7=f($ Time $)$; LS7 = f(S7); S8 = f(Time); LS8 = f(S8);S9 = f(Time $)$ LS9 = $f(S 9) ; S 10=f($ Time $) ; L S 10=f(S 10) ; L S=f(L S 1, L S 2, L S 3$, LS4, LS5, LS6, LS7, LS8, LS9, LS10).

$S 1$ represents the proportion of the community have a high school within a range of 1000 meters, LS1 represents the proportion livability of the community have a high school within a range of 1000 meters, S2 represents the proportion of the community have a junior high school within a range of 500 meters, LS2 represents the proportion livability of the community have a junior high school within a range of 500 meters, S3 represents the lighting facilities intact rate, LS3 represents the livability of lighting facilities intact rate, $S 4$ represents the urban water pipeline coverage rate, LS4 represents the livability of urban water pipeline coverage rate, S5 represents the urban biomass-gobbling popularizing rate, LS5 represents the livability of urban biomass-gobbling popularizing rate, $S 6$ represents the urban planning land for commercial financial center, LS6 represents the livability of urban planning land for commercial financial center, $S 7$ represents the every million peoples have leisure square area, $L S 7$ represents the livability of every million peoples have leisure square area, $S 8$ represents the every thousand peoples have hospital beds, LS8 represents the livability of every thousand peoples have hospital beds, $S 9$ represents the pipeline facilities intact rate, LS9 represents the livability of pipeline facilities intact rate, $S 10$ represents the road facilities intact rate, $L S 10$ represents the livability of road facilities intact rate, $L S$ represents the basic service facilities livability.

\section{F. Information service facilities livability fuction expressions}

The information service facilities livability fuction expressions include: $M 2=\mathrm{F}($ Time $) ; L M 2=f(M 2) ; M 5=$ $f($ Time $) ; L M 5=f(M 5) ; M 6=f($ Time $) ; L M 6=f(M 6) ; L M=$ $f(L M 2, L M 5, L M 6)$.

M2 represents the Internet penetration rate, LM2 represents the livability of Internet penetration rate, M5 represents the telephone number of every one hundred people, LM5 represents the telephone number livability of every one hundred people, $M 6$ represents the computer number livability of every one hundred people, LM6 represents the computer number livability of every one hundred people, $L M$ represents the information service facilities livability.

\section{G. Traffic service facilities and public service facilities livability fuction expressions}

The traffic service facilities and public service facilities livability fuction expressions include: $M 1=f($ Time $) ; L M 1=$ $f(M 1) ; M 3=f($ Time $) ; L M 3=f(M 3) ; M 4=f($ Time $) ;$ LM4 = $f(M 4) ; L T=f(L M 1, L M 3, L M 4) ; I 3=f(L T, L M, L S)$.

$M 1$ represents the road area per capita, LM1 represents the livability of road area per capita, M3 represents the public traffic sharing rate, LM3 represents the livability of public traffic sharing rate, $M 4$ represents the every ten thousand people have public transport number, LM4 represents the livability of every ten thousand people have public transport number, $L T$ represents the traffic service facilities livability, I3 represents the public service facilities livability.

\section{H. Social civilization degree livability fuction expressions}

The social civilization degree livability fuction expressions include: $Q 1=f($ Time $) ; L Q 1=f(Q 1) ; Q 2=$ $f($ Time $) ; L Q 2=f(Q 2) ; Q 3=f($ Time $) ; L Q 3=f(Q 3) ; L Q=$ $f(L Q 1, L Q 2, L Q 3)$.

Q1 represents the harmonious community construction rate, $L Q 1$ represents the livability of harmonious community construction rate, $Q 2$ represents the urban education facilities matching rate, LQ2 livability of urban education facilities matching rate, Q3 represents the urban sports facilities matching rate, LQ3 represents the sports facilities in livability of urban sports facilities matching rate, $L Q$ represents the social civilization degree livability. 


\section{Social stability degree and social harmony degree livability fuction expressions}

The social stability degree and social harmony degree livability fuction expressions include: $X 1=f($ Time $) ; L X 1=$ $f(X 1) ; X 2=f($ Time $) ; L X 2=f(X 2) ; X 3=f($ Time $) ; L X 3=f(X 3)$; $X 4=f($ Time $) ; L X 4=f(X 4) ; X 5=f($ Time $) ; L X 5=f(X 5) ; X 6=$ $f($ Time $) ; L X 6=f(X 6) ; X 7=f($ Time $) ; L X 7=f(X 7) ; X 8=$ $f($ Time $) ; L X 8=f(X 8) ; L X=f(L X 1, L X 2, L X 3, L X 4, L X 5, L X 6$, $L X 7, L X 8) ; I 4=f(L X, L Q)$.

$X 1$ represents the criminal case an average annual decline rate, $L X 1$ represents the livability of criminal case an average annual decline rate, $X 2$ represents the land-lost farmers employment rate, $L X 2$ represents the livability of land-lost farmers employment rate, $X 3$ represents the security community compliance rate, $L X 3$ represents the livability of security community compliance rate, $X 4$ represents the security group compliance rate, $L X 4$ represents the livability of security group compliance rate, $X 5$ represents the new labor force employment rate, $L X 5$ represents the livability of new labor force employment rate, $X 6$ represents the public security case an average annual decline rate, $L X 6$ represents the livability of public security case an average annual decline rate, $X 7$ represents the labor employment training rate, $L X 7$ represents the livability of labor employment training rate, $X 8$ represents the all kinds of contradictions and disputes mediation rate, $L X 8$ represents the livability that all kinds of contradictions and disputes mediation rate, $L X$ represents the social stability degree livability, I4 represents the social harmony degree livability.

\section{J. Economic development level livability fuction expressions}

The economic development level livability fuction expressions include: $J 1=f($ Time $)$; $L J 1=f(J 1)$; $J 2=f($ Time $)$; $L J 2=f(J 2) ; J 3=f($ Time $) ; L J 3=f(J 3) ; J 4=f($ Time $) ; L J 4=$ $f(J 4) ; L X=f(L J 1, L J 2, L J 3, L J 4)$.

$J 1$ represents the per capita disposable income of urban residents, LJ1 represents the livability of per capita disposable income of urban residents, $J 2$ represents the an average annual growth rate of per capita disposable income of urban residents, $L J 2$ represents the an average annual growth rate livability of per capita disposable income of urban residents, $J 3$ represents the worker average wage an average annual growth rate, $L J 3$ represents the livability of worker average wage an average annual growth rate, J4 represents the per capita GDP, LJ4 represents the livability of per capita GDP, $L J$ represents the economic development level livability.

\section{K. Economic development potential and economic development degree livability fuction expressions}

The economic development potential and economic development degree livability fuction expressions include: $K 1=f($ Time $) ; L K 1=f(K 1) ; K 2=f($ Time $) ; L K 2=f(K 2) ; K 3=$ $f($ Time $) ; L K 3=f(K 3) ; K 4=f($ Time $) ; L K 4=f(K 4) ; K 5=$ $f($ Time $) ; L K 5=f(K 5) ; L K=f(L K 1, L K 2, L K 3, L K 4, L K 5) ; I 5$ $=f(L J, L K)$.

$K 1$ represents the output value of enterprises with foreign investment, $L K 1$ represents the livability of output value of enterprises with foreign investment, $K 2$ represents the science and technology contribution rate to the enterprise, $L K 2$ represents the livability of science and technology contribution rate to the enterprise, $K 3$ represents the efficient characteristic industry an average annual growth rate, $L K 3$ represents the livability of efficient characteristic industry an average annual growth rate, $K 4$ represents the high and new technology industry accounted for the proportion of gross value of industrial output, LK4 represents the livability of high and new technology industry accounted for the proportion of gross value of industrial output, $K 5$ represents the entrepreneurship support policy coverage rate, LK5 represents the livability of entrepreneurship support policy coverage rate, $L K$ represents the economic development potential livability, I5 represents the economic development degree livability.

\section{Cultural atmosphere livability fuction expressions}

The cultural atmosphere livability fuction expressions include: $C 1=f($ Time $) ; L C 1=f(C 1) ; C 2=f($ Time $) ; L C 2=$ $f(C 2) ; C 3=f($ Time $) ; L C 3=f(C 3) ; C 4=f($ Time $) ; L C 4=f(C 4)$; $C 5=f($ Time $) ; L C 5=f(C 5) ; L C=f(L C 1, L C 2, L C 3, L C 4$, LC5).

C1 represents the number of public library new books, $L C 1$ represents the livability of number of public library new books, $C 2$ represents the civilized unit compliance rate, $L C 2$ represents the livability of civilized unit compliance rate, $C 3$ represents the cultural relic preservation status, LC3 represents the livability of cultural relics preservation status, C4 represents the higher education enrollment rate, LC4 represents the livability of higher education enrollment rate, C5 represents the residents civilization activity participation rate, $L C 5$ represents the livability of residents civilization activity participation rate, $L C$ represents the cultural atmosphere livability.

\section{Cultural facilities and cultural rich degree livability fuction expressions}

The cultural facilities and cultural rich degree livability fuction expressions include: $Y 1=f($ Time $) ; L Y 1=f(Y 1) ; Y 2=$ $f($ Time $) ; L Y 2=f(Y 2) ; Y 3=f($ Time $) ; L Y 3=f(Y 3) ; Y 4=f($ Time $)$; $L Y 4=f(Y 4) ; Y 5=f($ Time $) ; L Y 5=f(Y 5) ; L Y=f(L Y 1, L Y 2$, $L Y 3, L Y 4, L Y 5) ; I 6=f(L C, L Y)$.

$Y 1$ represents the large and comprehensive sports venues, LY1 represents the livability of large and comprehensive sports venues, $Y 2$ represents the cable TV penetration, $L Y 2$ represents the livability of cable TV penetration, Y3 represents the movie theaters number per ten thousand people, LY3 represents the livability of movie theaters number per ten thousand people, $Y 4$ represents the public library books number per one hundred people, LY4 represents the livability of public library books number per one hundred people, Y5 represents the number of higher learning institutions and scientific research institutions, LY5 represents the number livability of higher learning institutions and scientific research institutions, $L Y$ represents the cultural facilities livability, I6 represents the cultural rich degree livability. 


\section{N. Total livability fuction expressions of urban environment}

The total livability fuction expressions of urban environment include: $E 1=f(I 1, I 2, I 3) ; E 2=f(I 4, I 5, I 6) ; L=$ $f(E 1, E 2)$.

E1 represents the natural material environment livability, $E 2$ represents the social cultural environment livability, $L$ represents the total livability of urban environment.

\section{PARAMETER ESTIMATION OF MODEL VARIABLES}

In the livability evaluation model of urban environment, according to the parameters input values, the corresponding livability evaluation result of urban environment can be calculated. There is a certain relationship between the variables and livability, through the analysis of the data between variables and livability, the function relationship between variables and livability can be obtained, this function relationship can describe the change regulation between variables and livability accurately.

\section{A. Livable utility and livable utility function}

In the decision process, "Utility" is usually used to describe the various feasible scheme whether the results satisfy the decision maker wishes. "Utility" is defined as: assume the feasible scheme that decide problem with multiple possible result $x$, on the basis of subjective desire and value tendency of decision makers, each result of decision makers have different value and function, the measurement that reflect the value and function of result $\mathrm{x}$ to decision makers is called utility, denoted as $u=u(x)$ [6].

In order to quantify the environmental indicators of urban environment, this paper introduced the concept of livable utility: livable utility is used to describe the urban environment livability with quantitative effectiveness index, and using the livable utility function to express it.

By using logistic curve model to fit the utility function, can get livable utility function is defined as: set $x$ is the result value of livable utility decision, and satisfy the good or bad assignment sort principle that bad things body has small assignment, set $u(x)=1 /\left(1 / M+a b^{x}\right)$, if the following conditions is satisfied, then utility function $u=u(x)$ is called livable utility function: (1) if $0<x \leq 1$, then $u(x)=1$ is satisfied; (2) if $0<x<+\infty$, then $\lim _{x \rightarrow+\infty} u(x)=1$ is satisfied.

Livable utility function $u=u(x)$ is the quantitative expression of the urban environment livability, can use it as a livability evaluation tool. To be sure, the building of livable utility function should meet the following assumptions: (1) the various influence factors of the urban environment are independent of each other, and the livable utility functions of multiple factors affecting can be superposition; (2) the livability only consider the urban environment evaluation of user, without consider the contribution of environment builder; (3) the influence factors of urban environment is expressed with $x$, the livable utility function is expressed with $u(x)$, and $M /(1+a M)$; (4) based on the needs of the practical problems, set $1 \% \leq x$ and $u(1 \%)=0.01$.

\section{B. Parameters calculation of livable utility function}

Based on the above analysis, logistic curve satisfy the changing rule of the mixed type of livable utility function, can use the logistic curve fitting on livable utility function. Therefore, we can make use of logistic regression analysis method of SPSS[7] to estimate the curve parameters of livable utility function in this paper.

First, make sure the livable quantitative relation between the livable utility function of the dependent variable and independent variables, and set up data file in the SPSS. The independent variables is level 4 indexes of evaluation index system of the urban environment, the dependent variable is livability, based on he standard values of the evaluation index system, on livability and to determine the quantitative relation between the four indexes. According to the livable quantitative relation set up the data file in SPSS.

Then, in the SPSS, choose Analyze (analysis), Regression (Regression analysis), and Curve Estimation (Curve Estimation process), and other functions, using SPSS to estimate the parameter value of livable utility function. When choosing Logistic, must input an upper bound value in the "Upper bound" box, the upper bound value must be greater than the maximum value of the dependent variable. When using SPSS to estimate the parameter value of livable utility function, the upper bound value is 1 .

Finally, according to the parameters estimation results of SPSS, the parameters of livable utility function can be calculated.

Improving the utilization ratio of the nature resources and harmless disposal rate of the living garbage has become one of the major issues to the modern science and technology development. In this paper, using living garbage recycling utilization ratio, as an example, show the method and process of livable utility function parameters estimation.

Set the living garbage recycling utilization ratio with $x(0<x<+\infty)$, and set the livable utility function of living garbage recycling utilization ratio with $u=1 /\left(1 / M+a b^{x}\right)$, $0<x<+\infty$, then the values that $x$ satisfies $u(x)$ as shown in table I.

TABLE I. LIVABLE UTILITY FUNCTION ASSIGNMENT

\begin{tabular}{|c|c|}
\hline $\boldsymbol{x}$ & $\boldsymbol{u}(\boldsymbol{x})$ \\
\hline 0.01 & 0.01 \\
\hline 0.50 & 0.60 \\
\hline 0.90 & 0.99 \\
\hline
\end{tabular}

According to the data in table 1 , set up data files in the SPSS, taking $\mathrm{x}=1$, after curve analysis can estimate the model parameters and the curve fitting diagram, as shown in table II and figure 1.

TABLE II. LIVABLE UTILITY FUNCTION PARAMETERS ESTIMATION

\begin{tabular}{|l|c|c|l|l|c|c|c|}
\hline \multirow{2}{*}{ Equation } & \multicolumn{4}{|c|}{ Model Summary } & \multicolumn{2}{c|}{$\begin{array}{c}\text { Parameter } \\
\text { Estimation }\end{array}$} \\
\cline { 2 - 8 } & $\boldsymbol{R \times R}$ & $\boldsymbol{F}$ & $\boldsymbol{d} \boldsymbol{f} \mathbf{d} \boldsymbol{d} \mathbf{2}$ & $\boldsymbol{S i g}$ & $\boldsymbol{a}$ & $\boldsymbol{b}$ \\
\hline Logistic & 1.00 & 18176.298 & 1 & 1 & 0.005 & 1117.2 & $3.291 \mathrm{E}-5$ \\
\hline
\end{tabular}




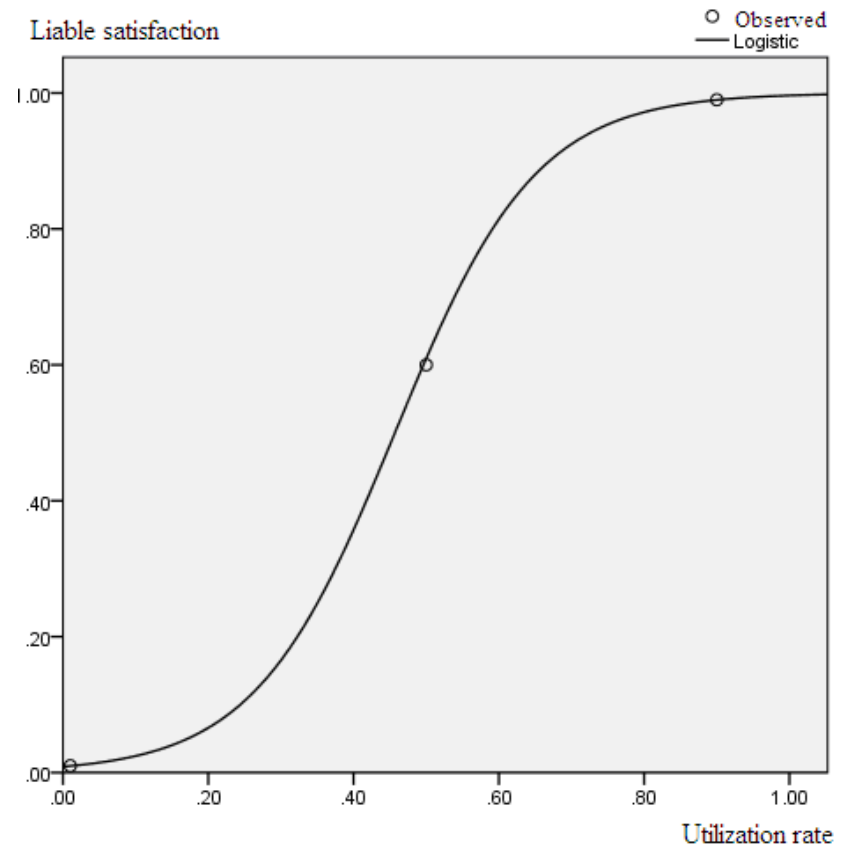

Figure 1. Living garbage recycling utilization ratio curve fitting chart

\section{Model VAlidation}

Through the simulation of livability evaluation model of urban environment can calculate the livability based on the different environment data, but also can detect the model further. The value of all model variables in this paper are derived from the statistical yearbook in the data, and are from 2001 to 2013 in the Tiexi district of Shenyang [4]. Through numerical endowed with different time period to the model variables, the corresponding numerical of livability can be calculated. As shown in table III.

TABLE III. LIVABILITY NUMERICAL ANNUAL DISTRIBUTION

\begin{tabular}{|c|c|}
\hline $\boldsymbol{x}$ & $\boldsymbol{u}(\boldsymbol{x})$ \\
\hline 2001 & 13.1 \\
\hline 2003 & 13.9 \\
\hline 2005 & 17.2 \\
\hline 2007 & 24.6 \\
\hline 2009 & 36.8 \\
\hline 2011 & 54.5 \\
\hline 2013 & 71.6 \\
\hline
\end{tabular}

The analysis result of the annual distribution of livability data shows, Tiexi district of Shenyang city, 2001-2005 is in the period of economic construction, development was more focused on the economy, the construction goal is in order to improve the material life of the residents demand, the attention to the urban environment is relatively small, so the growth rate of urban environment livability is very slow in 2001, 2003 and 2005.
With the rapid development of city construction, the problems such as housing shortage, environmental pollution, traffic jam, lack of resources becomes more and more outstanding. Facing the increasingly serious problem of city, how to build a livable urban, improve the quality of life of residents, improve the living environment, establish reasonable system of city ecological, realize the sustainable development of the urban, is the focus of attention of the residents and government. The livable attention to speed up the construction of urban environment. From the data in Table 3 can be seen, from the beginning of 2007, the increase of urban environment livability continues to accelerate, till 2013 urban environment livability reached $71.6 \%$.

Thus, the numerical of urban environment livability which obtained from livability evaluation model of urban environment, to a certain extent, is consistent whit the real life situation, then prove the effectiveness of the model.

\section{SUMMARY}

In this paper, through the research and analysis of the quantitative relationship between the urban environment factor evaluation index system, determine the main factors which impact the livability evaluation results of urban environment.

Using system analysis method to build livability evaluation system dynamics model of urban environment, and the parameter estimation method is used to determine the model variables. Combined with effective data provide statistical yearbook of Shenyang city Tiexi District, testing the effectiveness of the model with the method of system simulation, test results show that the model is effective.

\section{ACKNOWLEDGMENT}

This work was supported in part by a grant from the National 12th Five-Year Support Program Subject Project Foundation (2011BAJ06B04).

\section{REFERENCES}

[1] L.Y. Wu, Introduction to Science of Human Settlements. China Architecture \& Building Press, Beijing 2001.

[2] S.X. Lu, Q.Z. Qin. Research on Livable City Theory: submitted to Journal of Beijing City University(2012), p. 13-14.

[3] F.X. Hu, X.J. Hu. Construction on evaluation index system of urban livability: submitted to Journal of Ecological Economy(2014), p. 4244.

[4] Statistical Bureau of Shenyang Tiexi District, Tiexi statistical yearbook(2001-2013).

[5] Q.F.Wang. The Complex System Modeling Theory, Method and Technology(Science Press, Beijing 2011).

[6] Y. Zhang. Fundamentals of Operations. Tsinghua University Press, Beijing 2010.

[7] S. Wu, F.M. Pan, Statistical Analysis of SPSS Daquan. Tsinghua University Press, Beijing 2014. 